Proceedings of the Institute of Mathematics and Mechanics,

National Academy of Sciences of Azerbaijan

Volume 45, Number 2, 2019, Pages 205-212

https://doi.org/10.29228/proc.4

\title{
ON THE MAHLER HYPOTHESIS
}

\author{
ILGAR SH. JABBAROV AND GUNAY K. HASANOVA
}

\begin{abstract}
In the present paper we offer a new approach for proving the Mahler Hypothesis. This Hypothesis was first proved by V. G. Sprindzuk by his method of essential and inessential domains. It is best known that this question is equivalent to the statement that Diophantine exponent is $1 / n$ for almost every point on the variety $M=\left(x, x^{2}, \ldots, x^{n}\right)$. We show that it is possible to derive the result of Sprindzuk from the theorems on convergence exponent of special integral in Tarry's problem.
\end{abstract}

\section{Introduction}

In 1932, K. Mahler [8-9] introduced a new classification of transcendental numbers and investigated so-called by him S-numbers. He proved that almost all transcendental numbers are S-numbers. He formulated a hypothesis on the basic properties of these numbers. In the fundamental works [10-11] V. G. Sprindzuk proved Mahler's hypothesis and formulated new problems awaiting for their solutions. To formulate the main problems let us introduce basic notions.

Denote by $\Pi$ the following set of polynomials with integral coefficients of degree not exceeding $n$ :

$$
\Pi=\left\{f(x)=\sum_{i=0}^{n} a_{i} x^{i} \mid a_{i} \in Z\right\} .
$$

The number

$$
h(f)=\max \left(\left|a_{0}\right|,\left|a_{1}\right|, \ldots,\left|a_{n}\right|\right)
$$

is called to be the height of the polynomial $f(x)$. Let we are given with real transcendental number $\alpha$ (consequently $\alpha$ cannot be a root of any polynomial from $\Pi$ ). Let $h>0$ be a real number. Mahler showed that there is a constant $\kappa>0$ for which the inequality

$$
|f(\alpha)|>h^{-n \kappa} ; h=h(f)
$$

is satisfied for any polynomial $f(x)$ with the height $\leq h$ for almost all real numbers. He had found the value $\kappa=4+\varepsilon(\varepsilon>0$ is any). Mahler conjectured that it is possible to take $\kappa=1+\varepsilon$.

2010 Mathematics Subject Classification. 11K60, 11J83, 11J17.

Key words and phrases. Diophantine approximation, algebraic variety, extremal variety, convergence exponent. 
For a given real number $h>0$ we consider such polynomials for which heights doesn't exceed $h$ (it is clear that the number of such polynomials is finite). Denote by $\omega_{n}(\alpha)$ the supremum of that positive numbers $\gamma>0$, for which the inequality

$$
|f(\alpha)|<h^{-\gamma} ; h=h(f)
$$

is satisfied for infinite number of polynomials from $\Pi$, when $h \rightarrow \infty$. It means that for arbitrary $\varepsilon>0$ there is a non-bounded from above sequence $h_{1}, h_{1}, \ldots$ such that (1.1) is satisfied for all such $h_{m}$ with

$$
\gamma=\omega_{n}(\alpha)+\varepsilon
$$

This number is defined for every given $n$, and, by this reason one can define the number (finite or infinite)

$$
g=\varlimsup_{n \rightarrow \infty} \frac{\omega_{n}(\alpha)}{n} .
$$

Note that for transcendental numbers due to Dirichlet's principle we always have $\omega_{n}(\alpha) \geq n$ and therefore, $g \geq 1$. The Mahler hypothesis is consisted in the statement that $\omega_{n}(\alpha)=n$ for almost all transcendental numbers $\alpha$. By Khintchine's Transference Principle [3, p.100], this hypothesis is equivalent to the hypothesis on extremality of the variety $\left(x, x^{2}, \ldots, x^{n}\right)$.

In 1993, A. A. Karatsuba advanced an opinion that the question on extremality of some algebraic varieties could be investigated by using of results on convergence exponent in Tarry's problem. In the present work we show that this proposition is valid. So, we prove the result of Sprindzuk by a new method.

\section{Auxiliary results}

The following result is a variant of Borel-Cantelly's lemma and plays an important role in the questions concerning extremality of algebraic varieties (see[6]).

Lemma 2.1. Let $A_{q}(q=1,2, \ldots)$ be a sequence of measurable sets in $\mathbb{R}^{n}$, and

$$
\sum_{\mathrm{q}=1}^{\infty} \operatorname{mes} A_{q}<\infty .
$$

Then the measure of such real numbers which fall into infinite number of sets $A_{q}$ is zero.

Proof. Let's designate $E \subset \mathbb{R}^{n}$ the subset of that points $\bar{x} \in \mathbb{R}^{n}$ which is an element of infinitely many sets $A_{q}$. Then for every natural number $q$ and point $\bar{x} \in E$

$$
\bar{x} \in \bigcup_{m=q+1}^{\infty} A_{m} .
$$

So,

$$
E \subset \bigcup_{m=q+1}^{\infty} A_{m},
$$

which means that

$$
\mu(E) \leq \sum_{m=q+1}^{\infty} \mu\left(A_{m}\right)
$$


Since the series of the lemma is convergent then the sum above is less than arbitrary positive number $\varepsilon>0$. So, the set $E \subset \mathbb{R}^{n}$ really has a zero measure. Lemma 2.1 has been proven.

Our main auxiliary tool is Kavaleuskaya's lemma (see $[2,6,10]$ ).

Lemma 2.2. Let $q$ be a natural number, $f_{j}(\bar{x}), j=1, \ldots, N$ be a family of real measurable functions defined in the cube $\Omega=[0,1]^{r}$. Denote by $\mu(q)$ the measure of a set of that $\bar{x} \in \Omega=[0,1]^{r}$ for which

$$
\left\|f_{j}(\bar{x})\right\|<q^{-r_{j}}(1 \leq j \leq N) .
$$

Then,

$$
\mu(q)<<q^{-r} \sum_{\left|c_{1}\right|<q^{r_{1}}} \cdots \sum_{\left|c_{N}\right|<q^{r_{N}}}\left|\int_{\Omega} e^{2 \pi i\left(c_{1} f_{1}(\bar{x})+\cdots+c_{N} f_{N}(\bar{x})\right)} d \bar{x}\right| ;
$$

here $r=r_{1}+\cdots+r_{N}$, and the constant in the symbol $<<$ depends on $N$ only.

The proof of lemma 2.2 can be found in $[2,6]$. Our next auxiliary tool is a result on the convergence exponent of the special integral of Tarry's problem (see $[1,4])$. The number $\gamma$ is called to be the convergence exponent for the integral

$$
\int_{-\infty}^{\infty} \int_{-\infty}^{\infty} \cdots \int_{-\infty}^{\infty}\left|\int_{0}^{1} e^{2 \pi i\left(\alpha_{n} x^{n}+\alpha_{n-1} x^{n-1} \cdots+\alpha_{1} x\right)} d x\right|^{2 k} d \alpha_{n} d \alpha_{n-1} \cdots d \alpha_{1},
$$

if it converges when $2 k>\gamma$ and diverges when $2 k<\gamma$.

Lemma 2.3. We have $\gamma=1+n(n+1) / 2$ for the integral $(2.1)$.

Proof of this lemma is given in [1].

\section{Basic results}

The aim of our work is to prove the following theorem.

Theorem 3.1. For almost all transcendental numbers the equality $\omega_{n}(\alpha)=n$ is satisfied.

Proof. Note that if the inequality (1.1) is satisfied for some $\alpha$ and polynomial

$$
f(\alpha)=a_{k} \alpha^{k}+\cdots+a_{1} \alpha+a_{0},
$$

with the height $h(f) \leq q$ then the following inequality is satisfied for the polynomial $\varphi(x)=f(x)-a_{0}$ at the point $\alpha$ :

$$
\|\varphi(\alpha)\|<h^{-\gamma}
$$

It is sufficient to consider transcendental numbers from the interval $[0,1]$. The method applied in the work can be used for any unite segment on the real axes with integral end points.

Take in the lemma 2.2

$$
f_{1}(\bar{x})=f\left(x_{1}\right)+f\left(x_{2}\right)+\cdots+f\left(x_{K}\right)-f\left(x_{K+1}\right)-f\left(x_{K+2}\right)-\cdots-f\left(x_{2 K}\right),
$$


where $K$ is a natural number specified below, $\bar{x}=\left(x_{1}, x_{2} \ldots, x_{2 K}\right) \in[0,1]^{2 K}$, $f(x)=\sum_{i=0}^{n} a_{i} x^{i}, r=n+\delta,(\delta$ is arbitrarily small $), N=1$. Let $q$ be a natural number. Denote by $\mu(q)$ the measure of a set of such $\alpha \in[0,1]$ for which

$$
\|f(\alpha)\|<(2 K)^{-1} q^{-n-\delta} .
$$

Further, let us denote by $\mu_{0}(q)$ the measure of a set of that $\bar{\alpha}=\left(\alpha_{1}, \alpha_{2} \ldots, \alpha_{2 K}\right)$ $\in[0,1]^{2 K}$ for which

$$
\left\|f_{1}(\bar{\alpha})\right\|<q^{-n-\delta} .
$$

Now one can easily observed that when (3.1) satisfied for transcendental numbers $\alpha_{1}, \alpha_{2} \ldots, \alpha_{2 K}$ then (3.2) satisfied for $\bar{\alpha}=\left(\alpha_{1}, \alpha_{2} \ldots, \alpha_{2 K}\right)$, due to inequality

$$
\begin{gathered}
\left\|f\left(x_{1}\right)+f\left(x_{2}\right)+\cdots+f\left(x_{K}\right)-f\left(x_{K+1}\right)-f\left(x_{K+2}\right)-\cdots-f\left(x_{2 K}\right)\right\| \leq \\
\leq\left\|f\left(x_{1}\right)\right\|+\left\|f\left(x_{2}\right)\right\|+\cdots+\left\|f\left(x_{K}\right)\right\|+ \\
+\left\|f\left(x_{K+1}\right)\right\|+\left\|f\left(x_{K+2}\right)\right\|+\cdots+\left\|f\left(x_{2 K}\right)\right\| \leq q^{-n-\delta} .
\end{gathered}
$$

So, if we write

$$
A_{q}=\left\{\alpha \in[0,1] \mid\|f(\alpha)\|<(2 K)^{-1} q^{-n-\delta}\right\}
$$

then

$$
A_{q}^{2 K} \subset B_{q}=\left\{\bar{\alpha} \in[0,1]^{2 K} \mid\left\|f_{1}(\bar{\alpha})\right\|<q^{-n-\delta}\right\} .
$$

Therefore, separating the summand corresponding to the value $c=0$, we have $\mu^{2 K}\left(A_{q}\right) \leq \mu_{0}\left(B_{q}\right)$, and in consent with the lemma 2.2

$$
\begin{gathered}
\mu^{2 K}\left(A_{q}\right) \leq \mu_{0}\left(B_{q}\right)<<q^{-n-\delta}+q^{-n-\delta} \times \\
\times \sum_{|c|<q^{n+\delta}}\left|\int_{[0,1]^{2 K}} e^{2 \pi i c\left(f\left(\alpha_{1}\right)+\cdots+f\left(\alpha_{K}\right)-f\left(\alpha_{K+1}\right)+\cdots+f\left(\alpha_{2 K}\right)\right)} d \alpha_{1} \cdots d \alpha_{2 K}\right|
\end{gathered}
$$

and the constant in the symbol $<<$ depends on $\delta$ only. We can represent the integral on the last chain of the previous inequality (when $c \neq 0$ ) as follows:

$$
\begin{gathered}
\int_{[0,1]^{2 K}} e^{2 \pi i c\left(f\left(\alpha_{1}\right)+\cdots+f\left(\alpha_{K}\right)-f\left(\alpha_{K+1}\right)-\cdots-f\left(\alpha_{2 K}\right)\right)} d \alpha_{1} \cdots d \alpha_{2 K}= \\
=\left|\int_{[0,1]^{K}} e^{2 \pi i c\left(\varphi\left(\alpha_{1}\right)+\cdots+\varphi\left(\alpha_{K}\right)\right)} d \alpha_{1} \cdots d \alpha_{2 K}\right|^{2} \cdot
\end{gathered}
$$

Using a view of the polynomial one has:

$$
\begin{gathered}
\varphi\left(\alpha_{1}\right)+\cdots+\varphi\left(\alpha_{K}\right)=a_{n} \alpha_{1}^{n}+\cdots+a_{1} \alpha_{1}+\cdots+ \\
+a_{n} \alpha_{K}^{n}+\cdots+a_{1} \alpha_{K}=a_{n}\left(\alpha_{1}^{n}+\cdots+\alpha_{K}^{n}\right)+\cdots+ \\
+a_{1}\left(\alpha_{1}+\cdots+\alpha_{K}\right) .
\end{gathered}
$$

Therefore,

$$
\begin{aligned}
& \int_{[0,1]^{K}} e^{2 \pi i c\left(\varphi\left(\alpha_{1}\right)+\cdots+\varphi\left(\alpha_{K}\right)\right)} d \alpha_{1} \cdots d \alpha_{2 K}= \\
= & \int_{0}^{1} \cdots \int_{0}^{1} e^{2 \pi i\left(c a_{1} u_{1}+\cdots+c a_{n} u_{n}\right)} d \alpha_{1} \cdots d \alpha_{K} ;
\end{aligned}
$$


here we introduced the notations $u_{j}=\alpha_{1}^{j}+\alpha_{2}^{j}+\cdots+\alpha_{K}^{j}, j=1, \ldots, n$. Applying the consequence of lemma 1 from the work $[2,3]$, we can represent the last integral as follows:

$$
\begin{gathered}
\int_{0}^{1} \cdots \int_{0}^{1} e^{2 \pi i\left(c a_{1} u_{1}+\cdots+c a_{n} u_{n}\right)} d \alpha_{1} \cdots d \alpha_{K}= \\
\int_{0}^{K} \cdots \int_{0}^{K}\left(\int_{\Pi} \frac{d s}{\sqrt{G}}\right) e^{2 \pi i\left(c a_{1} u_{1}+\cdots+c a_{n} u_{n}\right)} d u_{1} \cdots d u_{n}
\end{gathered}
$$

where $\Pi$ denotes the surface defined by the system of equations

$$
u_{j}=\alpha_{1}^{j}+\alpha_{2}^{j}+\cdots+\alpha_{K}^{j}, j=1, \ldots, n,
$$

and $G$ is a Gram determinant of the gradients of functions $u_{j}$, i. e.

$$
G=\operatorname{det}\left(A A^{t}\right)
$$

and $A$ is a Jacoby matrix of the functions $u_{j}$ :

$$
A=\left(\begin{array}{cccc}
n u_{1}^{n-1} & n u_{2}^{n-1} & \cdots & n u_{K}^{n-1} \\
(n-1) u_{1}^{n-2} & (n-1) u_{2}^{n-2} & \cdots & (n-1) u_{K}^{n-2} \\
\vdots & \vdots & \ddots & \vdots \\
1 & 1 & \cdots & 1
\end{array}\right)
$$

Note also that the surface integral

$$
g(\bar{u})=\int_{\Pi} \frac{d s}{\sqrt{G}},
$$

for given $\bar{u}=\left(u_{1}, \ldots, u_{K}\right)$, is defined in improper meaning as a limit

$$
\lim _{\lambda \rightarrow 0} \int_{\Pi, G \geq \sqrt{\lambda}} \frac{d s}{\sqrt{G}},
$$

which defines a summable function of $\bar{u}$. Since the Gram determinant (3.4) can be represented as a sum of squares of all possible minors of maximal order of the matrix $A$, then

$$
G=\operatorname{det}\left(A A^{t}\right)=n ! \sum_{1 \leq i_{1}<\ldots<i_{n} \leq K} \prod_{1 \leq r<s \leq K}\left(u_{i_{r}}-u_{i_{s}}\right)^{2}
$$

because every of minors is a Vandermonde determinant multiplied by $n$ !. The integral at the right side of the equality (3.3) we can split into the sum of integrals taken over unite cubes:

$$
\begin{gathered}
\int_{0}^{K} \cdots \int_{0}^{K} g(\bar{u}) e^{2 \pi i\left(c a_{1} u_{1}+\cdots+c a_{n} u_{n}\right)} d u_{1} \cdots d u_{n}= \\
=\sum_{0 \leq j_{1}, \ldots, j_{n} \leq K-1} \int_{j_{1}}^{j_{1}+1} \cdots \int_{j_{n}}^{j_{n}+1} g(\bar{u}) e^{2 \pi i\left(c a_{1} u_{1}+\cdots+c a_{n} u_{n}\right)} d u_{1} \cdots d u_{n} .
\end{gathered}
$$

Each integral at the right side of this equality serves as a Fourier coefficient

$$
g_{c a_{1}, \ldots, c a_{n}}\left(j_{1}, \ldots, j_{n}\right)=\int_{j_{1}}^{j_{1}+1} \cdots \int_{j_{n}}^{j_{n}+1} g(\bar{u}) e^{2 \pi i\left(c a_{1} u_{1}+\cdots+c a_{n} u_{n}\right)} d u_{1} \cdots d u_{n}
$$

of the function represented by the corresponding part of the surface integral

$$
\int_{\Pi} \frac{d s}{\sqrt{G}}
$$


From (3.3) and (3.4) we get

$$
\begin{gathered}
\mu^{2 K}\left(A_{q}\right) \leq \mu_{0}\left(B_{q}\right) \leq q^{-n-\delta}+ \\
q^{-n-\delta} \sum_{0<|c|<q^{n+\delta}}\left|\sum_{0 \leq j_{1}, \ldots, j_{n} \leq K-1} g_{c a_{1}, \ldots, c a_{n}}\left(j_{1}, \ldots, j_{n}\right)\right|^{2} \leq \\
\leq q^{-n-\delta}+q^{-n-\delta} K^{n} \sum_{0<|c|<q^{n+\delta}} \sum_{0 \leq j_{1}, \ldots, j_{n} \leq K-1}\left|g_{c a_{1}, \ldots, c a_{n}}\left(j_{1}, \ldots, j_{n}\right)\right|^{2} .
\end{gathered}
$$

Inequality (3.5) we will use for the proof of our theorem. Denoting $c a_{m}=b_{m}$, transform the last sum at the right side of the inequalities (3.5). At first we note that due to constraints over the indexes we have $\left|b_{m}\right| \leq q^{n+1}$. So, the number of solution of the equation $x y=b_{m}$ in integral numbers doesn't exceed the number of divisors of $b_{m}$, multiplied by 4 i. e. the value $4 \tau\left(b_{m}\right)<<_{\varepsilon} q^{\varepsilon}$ for any positive $\varepsilon>0$, and the constant under the sign of the symbol $<<$ depends on $\varepsilon$ only. We have:

$$
\begin{gathered}
\mu^{2 K}\left(A_{q}\right) \leq \mu_{0}\left(B_{q}\right) \leq q^{-n-\delta}+ \\
q^{-n-\delta} K^{n} \sum_{0 \leq j_{1}, \ldots, j_{n} \leq K-1} \sum_{0<|c|<q^{n+\delta}}\left|g_{c a_{1}, \ldots, c a_{n}}\left(j_{1}, \ldots, j_{n}\right)\right|^{2} \leq \\
<<q^{-n-\delta}+q^{-n-\delta} K^{n} \sum_{0 \leq j_{1}, \ldots, j_{n} \leq K-1} \sum_{0<\left|b_{j}\right|<q^{n+1+\delta}, j=1, \ldots, n}\left|g_{b_{1}, \ldots, b_{n}}\left(j_{1}, \ldots, j_{n}\right)\right|^{2} .
\end{gathered}
$$

This inequality is valid for given polynomial $f$ with the height $h(f) \leq q$ and a given transcendental number $\alpha$. If we take all of Fourier coefficients, with the multiplicity indicated above for every of them, then we will increase the main sum. So, we have:

$$
\begin{gathered}
\mu_{0}\left(B_{q}\right) \leq q^{-n-\delta+\varepsilon}+q^{-n-\delta+\varepsilon} K^{n} \sum_{0 \leq j_{1}, \ldots, j_{n} \leq K-1} 1 \times \\
\times \sum_{\left|b_{1}\right| \leq q^{n+2}} \ldots \sum_{\left|b_{n}\right| \leq q^{n+2}}\left|g_{b_{1}, \ldots, b_{n}}\left(j_{1}, \ldots, j_{n}\right)\right|^{2} .
\end{gathered}
$$

Denote by $U_{q}$ the set of such transcendental numbers for which the inequality (1.1) is valid for some polynomial with the height $h(f) \leq q$. Then summing both sides of the got above inequality, from (3.6) we derive:

$$
\begin{aligned}
& \mu\left(U_{q}\right)<< q^{-\delta+\varepsilon}+q^{-n-\delta+\varepsilon} K^{n} \sum_{\left|a_{1}\right| \leq q} \ldots \sum_{\left|a_{n}\right| \leq q} \sum_{0 \leq j_{1}, \ldots, j_{n} \leq K-1} 1 \times \\
& \times \sum_{\left|b_{1}\right| \leq q^{n+2}} \ldots \sum_{\left|b_{n}\right| \leq q^{n+2}}\left|g_{b_{1}, \ldots, b_{n}}\left(j_{1}, \ldots, j_{n}\right)\right|^{2} \leq \\
& \leq q^{-\delta+\varepsilon}+q^{-\delta+\varepsilon} K^{n} \sum_{0 \leq j_{1}, \ldots, j_{n} \leq K-1} \sum_{\left|b_{1}\right| \leq q^{n+2}} \ldots \sum_{\left|b_{n}\right| \leq q^{n+2}}\left|g_{b_{1}, \ldots, b_{n}}\left(j_{1}, \ldots, j_{n}\right)\right|^{2} .
\end{aligned}
$$

Appling Parseval identity we can write:

$$
\sum_{b_{1}=-\infty}^{\infty} \cdots \sum_{b_{n}=-\infty}^{\infty}\left|g_{b_{1}, \ldots, b_{n}}\left(j_{1}, \ldots, j_{n}\right)\right|^{2}=\int_{j_{1}}^{j_{1}+1} \cdots \int_{j_{n}}^{j_{n}+1}(g(\bar{u}))^{2} d u_{1} \cdots d u_{n} .
$$


Consequently, taking $\varepsilon=\delta / 2$, we get:

$$
\begin{gathered}
\mu\left(U_{q}\right)<<q^{-\delta / 2}+ \\
+q^{-\delta / 2} K^{n} \sum_{0 \leq j_{1}, \ldots, j_{n} \leq K-1} \int_{j_{1}}^{j_{1}+1} \cdots \int_{j_{n}}^{j_{n}+1}(g(\bar{u}))^{2} d u_{1} \cdots d u_{n} \leq \\
\leq q^{-\delta / 2}+q^{-\delta / 2} K^{n} \int_{0}^{K} \cdots \int_{0}^{K}\left(\int_{\Pi} \frac{d s}{\sqrt{G}}\right)^{2} d u_{1} \cdots d u_{n} .
\end{gathered}
$$

As it was shown in [4-5] the last integral is equal to the special integral (2.1) of Terry's problem

$$
\int_{0}^{\infty} \cdots \int_{0}^{\infty}\left|\int_{0}^{1} e^{2 \pi i\left(\alpha_{1} x+\cdots+\alpha_{n} x^{n}\right)} d x\right|^{2 K} d \alpha_{1} \cdots d \alpha_{n}
$$

multiplied by $(2 \pi)^{-n}$. According to the lemma 2.3 the special integral has a convergence exponent equal to $1+n(n+1) / 2$. Therefore, when $2 K>1+n(n+$ $1) / 2$, the last integral is a constant. So, we have

$$
\mu\left(U_{q}\right)<<q^{-\delta / 2},
$$

in consent with (3.7). Taking $q=2^{k}$ consider the union

$$
\bigcup_{k=1}^{\infty} U_{2^{k}}
$$

Now we recall that the set $U_{q}$ is a set of such transcendental numbers $\alpha \in[0,1]$ for which the inequality (1.1) is satisfied for polynomials from $\Pi$ with the height not exceeding $q$. Then,

$$
U_{q}=\bigcup_{f \in \Pi, h(f) \leq q} W_{f}
$$

where

$$
W_{f}=\left\{\alpha|| f(\alpha) \mid<q^{-n-\delta}\right\}
$$

Since the series

$$
\sum_{k=1}^{\infty} \mu\left(U_{2^{k}}\right) \leq \sum_{m=0}^{\infty} 2^{-m \delta / 2}
$$

converges, then by the lemma 2.1 the set of such $\alpha$ which falls into infinite number of subsets $W_{f}$ is equal to 0 . So, we have proven that the number of polynomials with the height $\leq q$ satisfying (3.2) is not bounded as $q \rightarrow \infty$ for the set of transcendental numbers of zero measure. Therefore, the statement is valid for the polynomials satisfying (3.1). The theorem now has been proven.

Acknowledgements. The authors express their gratitude to professor M. Bayramoglu for useful discussions concerning results of this article. 


\section{References}

[1] G. I. Arkhipov , A. A. Karatsuba, V. N. Chubarikov, Thoery of multiple Triqonometric Sums., M.:, Nauka, 1987.

[2] V.I. Bernik, E. I. Kavaleuskaya, Extremality property of some surfaces in $n$ dimensional Eucidean space, Matemat. Zametki, 15(2) (1974), 247-254.

[3] J.W.S. Cassels, An introduction to Diophantine Approximation. IIL, Moscow, 1961.

[4] I.Sh. Dzhabbarov, The exponent of convergence of a special integral in multidimensional problems Terry, Chebyshevskii Sbornik, 14(2) (2013), 74-103.

[5] I.Sh. Dzhabbarov, On an identity of Harmonic Analysis and its applications, Dokl. AN USSR, 314(5) (1990), 1052-1054.

[6] E.I. Kavaleuskaya, Simultaneously extremal manifolds., Matemat. Zametki, 41(1) (1987), 3-8.

[7] A. Khintschine, Uber eine Klasse linearer Diophantischer Approximationen, Circolo mat. Palermo 50 (1926), 175-195.

[8] K.Zur Mahler, Approximation der Exponentialfunktionen und des Logarithmus. I, II., J. reine und angew. Math. 166 (1932), 118-150.

[9] K. Mahler, Uber das Mass der Menge aller S-Zahlen. Math. Ann. 106 (1932), 131-139.

[10] V. G. Sprindzuk, Metric theory of Diophantine Approximations., M. Nauka, 1977.

[11] V. G. Sprindzuk, A proof of Mahler's conjecture on the measure of the set of Snumbers, Izvestiya Mathematics, 16(1) (1981), 21-40.

Ilgar Sh. Jabbarov

Ganja State University, Ganja, Azerbaijan.

E-mail address: ilgar_j@rambler.ru

Gunay K. Hasanova

Department of Mathematical Analysis, Ganja State University, Ganja, Azerbaijan.

E-mail address: gunay.hasanova@mail.ru

Received: October 21, 2018; Revised: June 21, 2019; Accepted: June 27, 2019 